\title{
INSOLVENZ
}

\section{Antrag rechtzeitig stellen!}

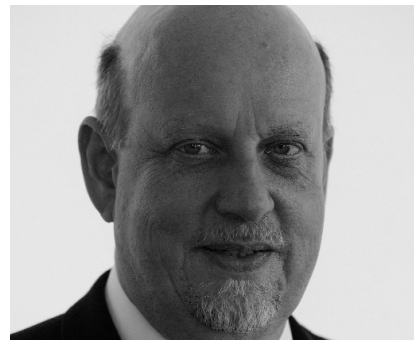

VON STEFAN SCHICK

Prof. Dr. Stefan Schick ist als Rechtsanwalt und Fachanwalt für Steuerrecht in Stuttgart tätig. Er ist seit langem Mitglied des Beirats der Zeitschrift SOZIALwirtschaft und des Informationsdienstes SOZIALwirtschaft aktuell.

E-Mail

Stefan.Schick@reithschick.de

\author{
Insolvenz ist auch in der Sozialwirtschaft kein Fremd- \\ wort mehr. Wenn eine soziale Organisation oder ein \\ sozialwirtschaftliches Unternehmen zahlungsunfähig \\ wird, haben die Verantwortlichen die gesetzliche \\ Pflicht, umgehend einen Insolvenzantrag zu stellen.
}

Ein unangenehmes Thema, über das man nicht gerne redet, das aber auch für Non-Profit-Organisationen zunehmend von Bedeutung ist: die Insolvenzantragspflicht - und den Folgen, die sich bei ihrer Verletzung für Vorstände und Geschäftsführer ergeben. Dabei gibt es Aspekte, die für Non-Profit-Organisationen in allen Rechtsformen gelten und solche, die rechtsformspezifisch sind.

\section{Für alle Rechtsformen geltende insolvenzrechtliche Grundlagen}

1.1. Zahlungsunfähigkeit, Überschuldung und drohende Zahlungsunfähigkeit als Insolvenzgründe: Eröffnungsgründe für ein Insolvenzverfahren sind nach $\mathbb{} 17$ Insolvenzordnung (InSo) die Zahlungsunfähigkeit, nach $\$ 18$ InsO die drohende Zahlungsunfähigkeit und nach $\mathbb{S} 19$ InsO die Überschuldung. Aber nur die Zahlungsunfähigkeit und die Überschuldung lösen die Insolvenzantragspflicht aus. Ein Schuldner ist dann zahlungsunfähig, wenn er nicht in der Lage ist, die fälligen Zahlungspflichten zu erfüllen ( $\int 17$ Abs. 2 InsO). Zahlungsunfähigkeit ist danach in der Regel anzunehmen, wenn der Schuldner seine Zahlungen eingestellt hat. Ein besonders wichtiges Indiz dafür ist es, wenn der Schuldner die Löhne und Gehälter nicht mehr bezahlt. Nach $\mathbb{S} 19$ Abs. 2 InsO liegt Überschuldung vor, wenn das Vermögen des Schuldners die bestehenden Verbindlichkeiten nicht mehr deckt (rechnerische Überschuldungsprüfung), es sei denn, die Fortführung des Unternehmens ist nach den Umständen überwiegend wahrscheinlich. Diese Regelung gilt in der genannten Form lediglich bis zum 31. Dezember 2013. Aufgrund des Finanzmarktstabilisierungsgesetzes und des Gesetzes zur Erleichterung der Sanierung von Unternehmen gilt ab dem 1. Januar 2014 wieder die alte Regelung: Überschuldung liegt dann vor, wenn das Vermögen des Schuldners die bestehenden Verbindlichkeiten (Rückstellungen und Verbindlichkeiten im bilanziellen Sinne) nicht mehr deckt. Bei der Bewertung des Vermögens des Schuldners ist jedoch die Fortführung des Unternehmens zugrunde zu legen, wenn diese nach den Umständen überwiegend wahrscheinlich ist.

1.2. Handlungsempfehlung für die Krise: Bis zum 31. Dezember 2013 ist zunächst zu prüfen, ob die Fortführung des Unternehmens nach den Umständen überwiegend wahrscheinlich ist. Ist dies der Fall, so hat keine Prüfung der rechnerischen Überschuldung stattzufinden. Dies setzt voraus, dass der Geschäftsführer oder Vorstand eine Ergebnis- und Liquiditätsplanung für das laufende und das Folgejahr aufstellt und sich daraus die positive Fortführungsprognose ableiten lässt. Im Rahmen der rechnerischen Überschuldungsprüfung sind die Aktiva und Passiva mit ihren Verkehrswerten anzusetzen, für den Zeitraum bis zum 31. Dezember 2013 mit ihren Liquidati- 
onswerten. Nach dem 31. Dezember 2013 muss immer eine rechnerische Überschuldungsprüfung durchgeführt werden, also geklärt werden, ob die Vermögensgegenstände die Schulden decken. Nur im Rahmen dieser rechnerischen Überschuldungsprüfung ist die Frage zu stellen, welchen Wert die einzelnen Vermögensgegenstände gegebenenfalls im Rahmen einer Fortführung des Unternehmens haben. Ferner sind bei einer negativen Fortführungsprognose in jedem Falle (also sowohl nach dem bis zum 31. Dezember 2013 als auch dem danach geltenden Recht) etwaige Verbindlichkeiten und Verpflichtungen $\mathrm{zu}$ berücksichtigen, die sich aus der Abwicklung ergeben, insbesondere Sozialplanansprüche.

\section{Rechtsformabhängige Pflichten}

2.1. Besonderheiten bei Non-ProfitOrganisationen in der Rechtsform der GmbH: Nach $\mathbb{S} 64$ GmbHG sind die Geschäftsführer der Gesellschaft zum Ersatz von Zahlungen verpflichtet, die nach Eintritt der Zahlungsunfähigkeit der Gesellschaft oder nach Feststellung ihrer Überschuldung geleistet werden. Dies gilt nicht für Zahlungen, die nach diesem Zeitpunkt erfolgen, aber mit der Sorgfalt eines ordentlichen Geschäftsmanns zu vereinbaren sind. Die Schadensersatzpflicht der Geschäftsführer wird durch ihre Verpflichtung zur Stellung des Insolvenzantrags in $\$ 15$ a Abs. 1 InsO ergänzt. Danach haben die Mitglieder des Vertretungsorgans einer juristischen Person oder die Abwickler ohne schuldhaftes Zögern, spätestens aber drei Wochen nach Eintritt der Zahlungsunfähigkeit oder Überschuldung, einen Insolvenzantrag zu stellen. Wird der Insolvenzantrag nicht, nicht richtig oder nicht rechtzeitig gestellt, so ist dies nach $\mathbb{S} 15$ a Abs. 4 InsO strafbar.

2.2. Besonderheiten bei Non-ProfitOrganisationen in der Rechtsform des eingetragenen Vereins und der rechtsfähigen Stiftung: Während die Überschuldung, die Zahlungsunfähigkeit und die drohende Zahlungsunfähigkeit auch bei eingetragenen Vereinen und rechtsfähigen Stiftungen Insolvenzgründe sind, gelten für die Haftung der Vorstände Besonderheiten. So sieht $\mathbb{S}$ 42 Abs. 2 Satz 2 BGB, auf den $\ 86$ BGB für die Stiftungen verweist, vor, dass der Vorstand, wenn er die Stellung des Antrags verzögert, den Gläubigern bei Verschulden haftet. Altgläubiger haben Anspruch auf den Betrag, um den sich die Insolvenzquote, die sie bei rechtzeitiger Antragstellung erhalten hätten, verringert. Neugläubiger haben gegen den schuldhaft handelnden Vorstand einen Anspruch auf Ersatz aller Schäden, die ihnen durch die Rechtsbeziehungen zu dem überschuldeten oder zahlungsunfähigen Verein entstehen. Im Gegensatz zur GmbH haften nach dem Beschluss des Bundesgerichtshofs vom 8. Februar 2010 (Az.: II ZR 54/09) die Vorstände aber nicht für Zahlungen, die nach der Insolvenzreife des Vereins erfolgen. Zwar gilt $\$ 15$ a InsO, nach dem der Insolvenzantrag unverzüglich, spätestens innerhalb von drei Wochen, zu stellen und die Verletzung dieser Frist strafbar ist, nach seinem Wortlaut auch für eingetragene Vereine und Stiftungen. Insoweit ist aber $\mathbb{} 42$ BGB, auf den $\$ 86$ BGB für Stiftungen verweist, als speziellere Regelung vorrangig. Dies bedeutet, dass die DreiWochen-Frist nicht gilt - der Insolvenzantrag ist unverzüglich zu stellen - und die Verletzung der Insolvenzantragspflicht nicht strafbar ist.

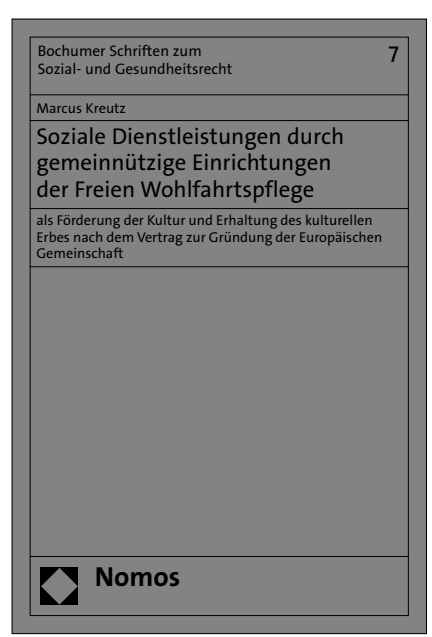

Soziale Dienstleistungen durch gemeinnützige Einrichtungen der Freien Wohlfahrtspflege

als Förderung der Kultur und Erhaltung des kulturellen Erbes nach dem Vertrag zur Gründung der Europäischen Gemeinschaft

Von RA Dr. Marcus Kreutz, LL.M.

2010, 362 S., brosch., 85,-€ ISBN 978-3-8329-5418-5

(Bochumer Schriften zum Sozialund Gesundheitsrecht, Bd. 7)

Nationale Privilegien, die der Gesetzgeber gemeinnützigen Körperschaften gewährt, sind nicht zwingend als verbotene Beihilfe im Sinne von Art. 87 Abs. 1 EGV zu qualifizieren. Vielmehr ergibt sich, dass diese Privilegien als Kultur und Erhaltung des kulturellen Erbes über Art. 87 Abs. 3 lit. d) EGV rechtfertigungsfähig sind.

\section{Nomos}

